\title{
GRUNDLAGEN FÜR PHONETISCHE ÜBUNGEN MIT ISLÄNDISCHSPRECHENDEN
}

\section{VORBEMERKUNG}

Isländisch ist ursprünglich ein westnorwegischer Dialekt, eine Einwanderersprache aus Norwegen, welche von den norwegischen Einwanderern, die in dem Zeitraum von 874 bis 930 n.Chr. Island besiedelten, ins Land gebracht wurde. Es waren sicherlich Einwanderer aus allen Gegenden Norwegens beteiligt, aber die meisten stammten aus Westnorwegen. Es gab ebenfalls eine Einwanderung aus Irland und den britischen Inseln. Es handelte sich dabei aber vorwiegend um norwegische Einwanderer die vorher zu den britischen Inseln und nach Irland eingewandert waren. Neben einigen irischen Einwanderern, die offensichtlich freiwillig emigriert sind, brachten die norwegischstämmigen Einwanderer zahlreiche irische Sklaven nach Island mit. Auf diese Weise erklärt es sich, daß etwa $30 \%$ der ursprünglichen Einwanderer in Island irischsprechend waren. Irisch wurde auch einige Zeit in Island gesprochen, obwohl es keine Spuren in der Sprache hinterlassen hat. Nur einige Orts- und Personenamen irischer Herkunft erinnern gegenwärtig an das ehemalige Vorhandensein der irischen Sprache in Island.

Bisher konnte nicht nachgewiesen werden, daß Island bereits vor der Ankunft norwegischer Einwanderer besiedelt war, obwohl einige Forscher dies stets behaupten. Somit ist Island das zuletzt besiedelte Land Europas im Mittelalter und die Geschichte Islands beginnt im Jahre 874 n.Chr.

Die Zeitspanne von 874 bis 930 wird die Landnahmezeit genannt. In dieser Zeit wurde das ganze Land besiedelt.

Mit der Gründung des Freistaates im Jahre 930 wurde die erste parlamentarische Demokratie Europas im Mittelalter gegründet. Der Freistaat bestand von 930 bis 1262 . Innere Streitigkeiten führten dazu, daß der norwegische König im Jahre 1262 einen Teil des Landes, und 1264 das ganze Land, unter seinen Einfluß bringen konnte. Island wurde norwegische Kolonie von 1262 bis 1380 . Im Jahre 1380 gelangte Norwegen unter dänische Herrschaft und somit auch Island. Von 1380 bis 1918 war Island dänische Kolonie. 1918 wurde Island ein autonomes Gebiet innerhalb des dänischen Königreiches, das nur durch die Person des Königs eine Verbindung zu Dänemark hatte. Im Jahre 1944 wurde Island schließlich mit der Gründung der Republik am 17. Juni 1944 erneut ein selbständiger Staat.

\subsection{Perioden der isländischen Sprachgeschichte}

Es ist üblich, die Geschichte der isländischen Sprache in vier sprachgeschichtliche Perioden einzuteilen:

1. Altisländisch (874-1150): In dieser Periode ist der Unterschied zum Norwegischen sehr gering. 
2. Klassisches Isländisch (1150-1350): In diesem Zeitraum entstanden alle Werke der berühmten mittelalterlichen Literatur, die Island in der europäischen Kulturgeschichte so bedeutsam gemacht haben. Auch wenn die ursprüngliche Grundlage einiger Werke bis in die Völkerwanderungszeit zurückgeht, wurden sie in diesem Zeitraum auf Pergament in Island niedergeschrieben. Gleichzeitig beginnt in diesem Zeitraum die Entwicklung sprachlicher Besonderheiten, die Isländisch und Norwegisch voneinander deutlich trennen.

3. Mittelisländisch (1350-1530): In diesem Zeitraum ereignen sich die wichtigsten Veränderungen, die Altisländisch und klassisches Isländisch von der modernen Sprache unterscheiden. Hierzu gehört in erster Linie die Aufgabe der segmentalen Quantität, welche durch eine Silbenquantität ersetzt wird. Als Folge davon ergeben sich bedeutsame Änderungen der Vokalqualität. Auch das Konsonantensystem ist betroffen, obwohl die Änderungen weniger bedeutsam sind.

4. Modernes Isländisch (1530-bis heute): Seit 1530 haben keine bedeutsamen Veränderungen mehr stattgefunden. Die Sprache hat sich seit damals kaum verändert.

Im 20. Jahrhundert zeichnet sich der Beginn einer neuen Instabilität in der Sprache ab. Hier sollen nur zwei Beispiele kurz erwähnt werden:

(1) In weiten Teilen der Bevölkerung wird die konsonantische Quantität aufgegeben und nur die Vokalquantität bleibt erhalten. Solche Änderungen haben vorher ebenfalls im Dänischen und Norwegischen stattgefunden. Auch das Deutsche ist eine Sprache, die nur Vokalquantität besitzt.

(2) Eine weitere Besonderheit der gesprochenen Sprache besteht in der enormen Verkürzung von Wörtern oder ganzen Äußerungen um einen Hauptakzent:

\begin{tabular}{|c|c|c|}
\hline Hvaõ segirðu? & Kvasiru? & „Was sagst Du?“ \\
\hline Maơur & mar & „Mann““ \\
\hline Hljómsveitaræfing & hljónstræng & „Orchesterübung“ \\
\hline Hljómsveit & hljónst & „Orchester" \\
\hline Forsætisráoherra & fostróðra & „Ministerpräsident ${ }^{6}$ \\
\hline Djóoleikhús & pólgús & "Nationaltheater" \\
\hline \multicolumn{2}{|c|}{ Hvao er petta? [ $\mathrm{k}^{\mathrm{h}} \mathrm{va}: ð$ er $\left.\theta \mathrm{ehta}\right]:$ katta $\left[\mathrm{k}^{\mathrm{h}} \mathrm{ahta}\right]$} & „Was ist das?“ \\
\hline
\end{tabular}

Diese Tendenz greift mit beängstigender Geschwindigkeit um sich und macht zusammengesetzte Wörter und ganze Äußerungen unanalysierbar. Nach vielen Jahrhunderten Stabilität (Grönke 1983) ist die Sprache auf dem Wege in eine neue Periode von Instabilität, die das Erscheinungsbild der Sprache binnen kurzem radikal ändern könnte.

\subsection{Sprecherzahl, geographisches Gebiet und sprachliche Einheit}

Isländisch wird von 307.000 Menschen in Island gesprochen. Darüberhinaus gibt es eine große Zahl von Isländischsprechenden in Dänemark, Schweden und Norwegen, insgesamt wohl etwa 10.000 Sprecher. In den Vereinigten Staaten von Amerika und in Kanada gibt es einige Tausende von Isländischsprechenden. Kleinere Gruppen von ihnen 
gibt es auch in Luxemburg, in Namibia, in Chile und in Australien, so daß ihre Zahl wahrscheinlich etwa 320.000 beträgt, eventuell sogar etwas darüber.

Island ist in letzter Zeit zu einem Einwanderungsland geworden. Laut der Statistik des Kultusministeriums von 1995 gibt es in den Grundschulen des Landes fremdsprachliche Schüler mit insgesamt 57 unterschiedlichen Muttersprachen. Schüler und Jugendliche lernen Isländisch schnell und häufig grammatisch völlig einwandfrei und ohne Fremdakzent. Dagegen fällt es älteren Einwanderern schwer, diese stark flektierte Sprache zu lernen.

Da Isländisch ursprünglich ein norwegischer Einwandererdialekt ist, gehört es zum nördlichen Zweig der skandinavischen Sprachen. Isländisch und Altnordisch sind die gleiche Sprache. Als Altnordisch wird im allgemeinen die Sprache der altnorwegischen und altisländischen Textausgaben bezeichnet. Zum Altisländischen besteht aber überhaupt kein Unterschied.

Das moderne Isländisch ist außerordentlich einheitlich in der Form. Dies ist auch der Fall mit anderen Einwanderersprachen, z.B. mit dem Englischen in Nordamerika und dem Spanischen in Südamerika. Im Isländischen ist der einheitliche Charakter allerdings noch stärker ausgeprägt. Es existieren überhaupt keine Dialekte. Es treten nur geringfügige Unterschiede in der Verteilung einiger Laute und in der Verwendung einiger Lexeme auf. Es gibt keine kodifizierte Norm des Isländischen und auch kein Aussprachewörterbuch. Soziologische Unterschiede gibt es kaum. Jede gesprochene Form des Isländischen ist akzeptabel. In diesem Sinne ist Isländisch sicher die einheitlichste Sprache Europas.

\section{ALLGEMEINE PHONETISCHE MERKMALE}

Da Isländisch eine germanische Sprache ist, ist kaum zu erwarten, daß es im Gegensatz zum Deutschen besondere auffällige phonetische Merkmale aufweist. Allerdings gibt es zwei phonetische Eigenschaften, die im Vergleich zu den festlandskandinavischen Sprachen auffällig sind:

1. Dem Isländischen fehlt jede Form prosodischer Akzentkorrelation. Die für das Schwedische und Norwegische so charakteristische Tonakzentuierung und der für das Dänische charakteristische Stoßton (,stød`) fehlen im Isländischen ganz.

2. Das Isländische hat eine unverhältnismäßig hohe Zahl stimmloser Konsonanten. Alle Verschlußlaute sind in allen Stellungen völlig stimmlos. Sie werden nur im betonten (bzw. nebenbetonten) Silbenanlaut durch die Aspiration unterschieden. In allen übrigen Stellungen sind nur die unaspirierten Verschlußkonsonanten vorhanden. Außerdem gibt es eine Stimmhaftigkeitskorrelation für Nasale, Laterale und Vibranten. Im Inlaut vor bestimmten Verschlußlauten taucht ein [h] auf, das in den Lautbeschreibungen häufig ,Präaspiration' genannt wird. Aus vielen Gründen, die ich ausführlich erläutert habe, empfiehlt es sich jedoch dieses Segment als den Konsonanten $[\mathrm{h}]$ zu betrachten und nicht als Teil des nachfolgenden Konsonanten (Pétursson 1972).

\section{SUPRASEGMENTALIA}

Bisher gibt es keine phonetische Untersuchungen zu den Suprasegmentalia im Isländischen. Die folgende kurze Beschreibung ist deshalb rein impressionistisch bzw. auditiv zu betrachten. 


\subsection{Akzent}

Alle Wörter, die im Isländischen verwendet werden, d.h. Wörter aus dem ursprünglichen Wortschatz, dem Erbwortschatz, und auch alle Fremdwörter, haben einen Hauptakzent auf der ersten Silbe (die betonte Silbe ist hier unterstrichen ( Hauptakzent, $=$ Nebenakzent)):

spennna „spannen“; hálfvirð̋ „halber Preis"; fangi „Gefangener"; alda „Welle; transistor „Transistor"

Wenn es sich um Zusammensetzungen handelt, wird der Hauptakzent auf die erste Silbe gelegt, aber die weiteren Komponenten erhalten sogenannte Nebenakzente, welche jedoch deutlich schwächer als der Hauptakzent sind:

bịllageymsla „Parkhaus"; landhelgi „Hoheitsgewässer“;

lágprýstingur „Niedrigdruck“; bókasafn „Bücherei“"

Diese Regel ist ausnahmslos und bringt wiederum den Beweis für die große Regelmäßigkeit der isländischen Akzentuierung.

Zum isländischen Akzent liegen zur Zeit keine phonetischen Untersuchungen vor. Auditiv hört er sich aber fast identisch oder identisch mit dem deutschen Akzent an.

\subsection{Intonation}

In Aussagesätzen ist die Intonation im Isländischen fallend. Sie fällt von der ersten betonten Silbe bis zu der letzten Silbe der Äußerung ab:

bú kemur á morgun. „Du kommst morgen"

In Fragesätzen ist die Intonation steigend:

Kemurðu á morgun? „Kommst Du morgen?“

Die Intonation kann alleine, ohne Änderung der Wortfolge, als Signal für die Frage funktionieren:

Dü kemur „Du kommst“ (Aussage)

bú kemur? „Du kommst?“ (Frage)

Untersuchungen zur Intonation im Isländischen fehlen zur Zeit ganz. Nicht einmal impressionistische Beschreibungen sind vorhanden. Dieses komplexe Gebiet harrt der Forschung. Über die Intonation als Gestaltungselement kommunikativer Funktion im Sinne von Selting (1995) ist wissenschaftlich nichts bekannt, obwohl jeder Sprecher ein solches Signalsystem unbewußt kennt und im Gespräch verwendet.

\subsection{Rhythmik}

Untersuchungen zur Rhythmik im Isländischen liegen zur Zeit nicht vor. Rein impressionistisch können jedoch folgende Besonderheiten hervorgehoben werden:

1. Quantitativ gesehen gibt es nur zwei Silbentypen:

/V:C/ langer Vokal + kurzer Konsonant

ala [a:la], ,ernähren“, tala [ $\left.\mathrm{t}^{\mathrm{b}} \mathrm{a}: \mathrm{la}\right]$,,sprechen",

bila [pi:la]"Panne haben", týna [ $\left.\mathrm{t}^{\mathrm{h}} \mathrm{i}: \mathrm{na}\right]$ "verlieren"

NC:/ kurzer Vokal + langer Konsonant bzw. Konsonantengruppe:

alla [atla] ,alle“ (Akk.Pl.), falla [fatla] „fallen“,

rabba [rap:a] ,quatschen, reden“, Ella [el:a] weiblicher Vorname

Auf diesen Aspekt der Quantität wird genauer unter 3.1. eingegangen. 
2. Eine Silbe, die auf eine betonte Silbe folgt, ist normalerweise die schwächste. Die zweite Silbe nach der betonten Silbe ist etwas stärker. Durch die Alternanz von Silben unterschiedlicher Stärke entsteht ein wellenartiger Rhythmus, der durch die folgenden Beispiele illustriert werden könnte ${ }^{1}$ :

$\mathrm{x} \mathrm{y} \mathrm{x}$

flutningunum ,den Transporten“ (Dat.Pl. mit suffigiertem Artikel)

$=\mathrm{x} \quad \mathrm{y}=\mathrm{x} \mathrm{y}$

húseigendafélagið „,der Hausbesitzerverein“

- $\mathrm{x}=\mathrm{x} \mathrm{y}=\mathrm{x} \quad \mathrm{y} \mathrm{x}$

barnaverdunarsampykktirnar „die Kinderschutzvereinbarungen“

$\mathrm{x}=\mathrm{x} \quad \mathrm{y} \quad \mathrm{x}=\mathrm{x} \mathrm{y}$

hafnarverkamannaverkföllin ,die Hafenarbeiterstreiks“

Diese rhythmischen Abstufungen ergeben sich nicht nur bei zusammengesetzten Wörtern wie in den obigen Beispielen, sondern auch in ganzen Sätzen:

' $\mathrm{x}{ }^{\prime} \mathrm{x} \mathrm{y}^{\prime} \mathrm{x}{ }^{\prime}=\mathrm{x} \quad \mathrm{y}{ }^{\prime} \mathrm{x} \quad \mathrm{y} \quad \mathrm{x}{ }^{\prime} \mathrm{x}$

Baendur héldu til fundar, vongódir um árangur, en urðu

$=\mathrm{x} \quad \mathrm{y} \times{ }^{\prime} \mathrm{x}=\mathrm{x} \quad \mathrm{y}$

sárvonsviknir með niðurstöðurnar

„Die Bauern kamen zum Treffen, hoffnungsvoll auf das Ergebnis, wurden aber durch die Resultate bitter enttäuscht."

\section{SEGMENTE}

\subsection{Silbenstruktur}

Im modernen Isländischen kommen zahlreiche Typen offener und geschlossener Silben vor. Im folgenden werden die wichtigsten Silbentypen aufgezählt:

\subsubsection{Offene Silben}

$N: / \quad a$, ,Fluß $\beta^{*}, i$, in $"$

/CV:/ $\quad f a ́$, ,erhalten“, vá „Unglück“, $k u$ „,Kuh“, sá „dieser“", $n u ́$, jetzt"

/CCV:/ frá „von“, froe „Samen“, slá „mähen, schlagen“"

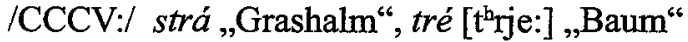

In offener betonter Silbe können nur lange Vokale oder lange Diphthonge stehen.

\subsubsection{Geschlossene Silben}

/V:Cl ár „Jahr“, án „ohne“, ól „Gürtel“"

NC:/ ann „(er) mag“, iss „Eis(es)“ (Gen.Sing.), egg „Ei“, enn „noch“

/CV:Cl $\quad k o l$, ,Kohle“, bar ,trug“(Prät. von bera ,tragen“), bol „Ausdauer“, bor „Bohrer“, búr „Käfig“, sár „Wunde“"

'Zeichenerklärung: '-(betonte Silbe), =(nebenbetonte Silbe), x (schwächste Silbe), y (etwas hervorgehobene Silbe) 
/CVC:/ sigg „Schwiele“, vegg „Wand“ (Akk.Sing.), senn „bald“, sinn „sein“ (Pronomen), vinn ,,(ich) arbeite“, vann ,,(er) arbeitete"(Prät. von vinna „arbeiten"), viss ,sicher", $k y r r$,ruhig“"

$/ \mathrm{CVCCC} /$ fengs "des Fanges, des Gewinns“ (Gen.Sing.)

/CCV:Cl frýs „es friert", frces „,des Samens“ (Gen.Sing.), brín „Kante“, snýr „,er dreht“", brýr „Brücken“ (Nom.P1.)

/CCVCCC/ drengs ,des Jungen“ (Gen.Sing.)

/CCCVCCC/strengs „,des Fadens“ (Gen.Sing.)

In der betonten offenen Silbe ist der Vokal immer lang. Ist die Silbe geschlossen, ist der Vokal kurz. Ausnahmen bilden nur geschlossene Silben mit Konsonantengruppen (Graphemen):

$$
\begin{aligned}
& <\text { kv kr }> \\
& <t v \text { tr } \mathrm{tj}\rangle \\
& <\text { sv } \text { sr sj }>
\end{aligned}
$$

vor denen der betonte Vokal lang bleibt:

vökva „,bewässern“, akra „Äcker“ (Akk.P1.), uppgötva ,entdecken“"

(Nebenakzent auf -götva), titra ,zittern“, etja ,anspornen“, hásra „heisere(n)“(Gen.P1.), Esja (Eigenname).

Allerdings muß vermerkt werden, daß die Vokale vor diesen Konsonantengruppen in der Umgangssprache häufig gekürzt werden. Dadurch werden diese Konsonantengruppen an andere Konsonantengruppen assimiliert, vor denen der Vokal immer kurz war.

\subsection{Vokale}

\subsubsection{Monophtonge}

Das Isländische hat acht Vokale (Monophtonge), die alle lang und kurz in betonter Silbe vorkommen. Im Vokaltrapez können Sie folgendermaßen dargestellt werden:

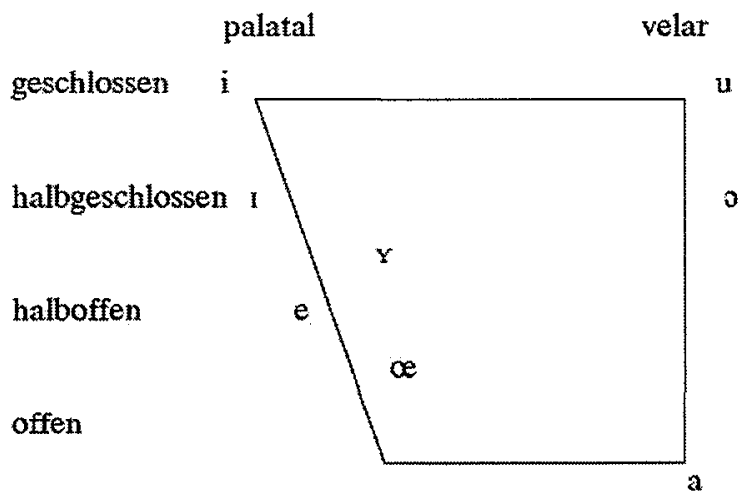


Die Vokale [y œ u o] sind gerundet. Die übrigen vier Vokale sind ungerundet. Zentrale Vokale kommen nicht vor.

Beispiele:

[i:] líta [li:ta] „schauen", $n y ́$ [ni:]"neu“, vik [vi:k] „kleine Bucht"

[i] býtta [pihta] ,austauschen“", nýtt [niht] ,neu“"

(Neutrum Sing. Nom.)

[r:] biti [pr:tr] „Happen“, bil [pr:1] ,Intervall“"

[I] bytta [prhta] „kleines Boot“, hitt [hiht] „getroffen“ (Part.Perf. von hitta „treffen")

[e:] betur [pe:tyr] „besser“, sem [se:m] „ich verfasse“ (Präs. von semja "verfassen")

[e] best [pest] ,am besten“, belja [pelja] ,Kuh“

[Y:] buna [py:na] „kleiner Wasserfall“", suða [sY:ða] „Kochen“, sumar [sY:mar] "Sommer“

[Y] sund [synt] „Schwimmen“, fundur [fyntyr] „Sitzung“"

[œ:] öl [œ:l] „Brause“, möl [mœ:l] „Schotter“

[œ] höll [hœtl] „Schloß“, völlur [vœtlyr] „Flachland, Fläche“

[u:] fús [fu:s] ,willig“, hús [hu:s] „Haus"

[u] kústur [k $\left.{ }^{\mathrm{h} u s t y r}\right]$ „Besen“, rüst [rust] „Ruine“"

[o:] koma [k ${ }^{\mathrm{h}}$ :ma] ,kommen“, $\log a[1 \mathrm{l}: \gamma \mathrm{a}]$,lodern, flammen“, svo [svo:] „so“"

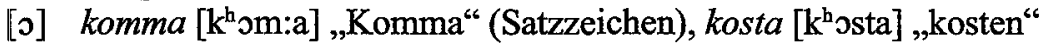



[a] kasta [k $\mathrm{k}^{\mathrm{h}}$ asta] ,werfen“, land [lant] „Land“

In unbetonter Silbe kommen in dem Grundwortschatz faktisch nur die Vokale i, u, a [ I Y a] vor, welche quantitativ neutral sind. Ausnahmen hierzu gibt es nur in zusammengesetzten Wörtern, in Eigennamen und in Fremdwörtern:

júní [ju:ni] „Juni“, júli [ju:li] „Juli“",Ellý [eli] (weiblicher Vorname),

kannske [ $\mathrm{k}^{\mathrm{h}}$ ansce] „vielleicht", máske [mausce] „vielleicht"

Die Vokale [œu o] kommen in unbetonter Silbe wahrscheinlich überhaupt nicht vor, wenigstens nicht im Auslaut.

\subsubsection{Diphthonge}

Die isländischen Diphthonge sind genau wie die Monophtonge in betonter Silbe lang oder kurz. Im Vokaltrapez können sie folgendermaßen schematisiert werden:

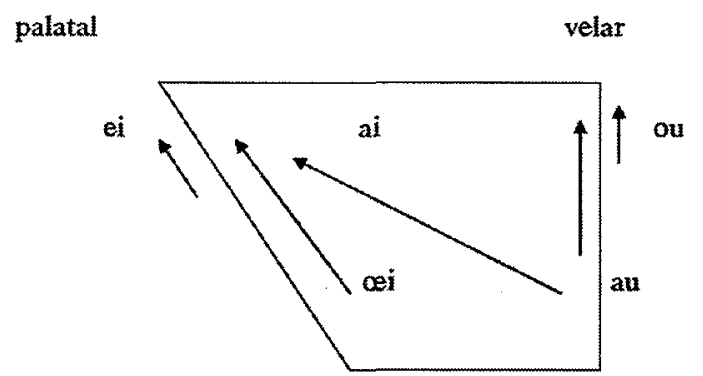


Die Pfeile geben die Bewegungsrichtung an. Die Diphthonge [ou au œi] sind durchweg gerundet, obwohl [a] und [i] als Monophthonge ungerundet sind.

[ei:] bein [pei:n] „Knochen“, sein [sei:n] „spät“ (Fem.Nom.Sing. von seinn ,spät")

[ei] einn [eitn] „ein", meistari [meistart] „Meister"

[ou:] ól [ou:1] „Gürtel“, sól [sou:1] „Sonne“, fór [fou:r] „fuhr"

[ou] ósk [ousk] „Wunsch“, brjósk [prjousk] „Knorpel““

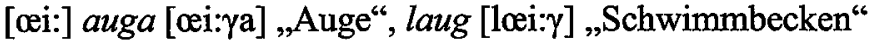

[œi] austur [œistYr] „Osten“"

[ai:] loeti [lai:tr] „Lärm“, loesa [lai:sa] „schließen“"

[ai] loest [laist] „geschlossen“, rcesta [raista] „reinigen“"

[au:] á [au:] „Fluß“, hás [hau:s] „heiser“, lá [lau:] „lag“ (Prät. von liggja „liegen“)

[au] ást [aust] „Liebe", fást [faust] „erhältlich sein“"

Diphthonge kommen in unbetonten Silben faktisch nur in Zusammensetzungen vor:

óró [ou:rou] „Unruhe“, bergvatnsá [perkvahtnsau] „Fluß“ (der kein Gletscherschmelzwasser fördert), Garðabcer [karðapair] (Ortsname)

Das zweite Element der Diphthonge ist etwas offener als die Vokale [i u], obwohl dieser Unterschied in der Transkription nicht sichtbar ist. Beim Diphthong [ou] ist die Bewegung der Zunge am geringsten. Meiner Meinung nach wäre es sogar möglich, hier ein geschlossenes [o] zu transkribieren, zumindest in der Aussprache einiger Sprecher.

\subsubsection{Monophtongierung der Diphthonge}

In der Umgangssprache macht sich die Tendenz bemerkbar, die kurzen Diphtonge zu monophtongieren. Es handelt sich aber nicht um eine allgemeine Tendenz, sondern um eine an gewisse Lexeme gebundene Tendenz. Hier ein Beispiel für jeden Diphthong:

\begin{tabular}{|c|c|c|c|c|}
\hline cetla & [aihtla] & $\rightarrow$ & [ahtla] & "vorhaben" \\
\hline stur & [œistYr] & $\rightarrow$ & [Ystyr] & "Osten" \\
\hline kólna & [k $\mathrm{k}^{\mathrm{h}}$ oulna $]$ & $\rightarrow$ & {$\left[\mathrm{k}^{\mathrm{h}} \mathrm{\rho lna}\right]$} & „kälter werden“ \\
\hline reyttur & [Oreihtyr] & $\rightarrow$ & {$[\theta$ rehtyr] } & "müde" \\
\hline hátt & [hauhta] & $\rightarrow$ & [hahta] & "ins Bett gehen" \\
\hline
\end{tabular}

Diese Tendenz kann dadurch erklärt werden, daß die artikulatorische Bewegung für die kurzen Diphthonge wesentlich geringer ist als für die langen (Pétursson 1974). Die Monophthongierung stellt daher eine Art Ökonomie der Sprechbewegungen dar.

\subsubsection{Bemerkungen zur Artikulation der Vokale}

Das isländische [e] liegt etwa in der Mitte zwischen dem deutschen [e] und $[\varepsilon]$. Es ist manchmal sehr schwierig für einen Isländer zwischen diesen beiden Vokalen zu unterscheiden.

Das isländische [œ] ist wesentlich offener als das deutsche [œ] in könnte; auch ist das isländische [Y] offener als das deutsche [Y] in Hütte.

Der Öffnungsgrad des isländischen [o] liegt etwa in der Mitte zwischen dem deutschen [o] in holen und dem [o] in sollte. 
Das isländische [a] wird trotz seiner relativ hellen Klangfarbe ziemlich weit nach hinten gebildet, auf jeden Fall weiter hinten als das deutsche [a] in hatte.

Schließlich muß der Deutsche, der Isländisch lernen möchte, sehr darauf achten, daß alle Klangfarben der Vokale (Monophthonge) und der Diphthonge lang und kurz vorkommen. Im Deutschen kommt eine Klangfarbe entweder kurz oder lang vor, mit der einzigen Ausnahme von [ع], das z.B. in hätte kurz, und in Ähre lang ist.

\subsection{Konsonanten}

Das Konsonantensystem des Isländischen kann in der folgenden Tabelle dargestellt werden:

\begin{tabular}{|l|c|c|c|c|c|c|}
\hline Artikulationsstelle & Bilabial & Labiodental & Dento-alveolar & Palatal & Velar & Glottal \\
\hline Artikulationsmodus & & & & & & \\
\hline Verschluß & $\mathrm{p} \mathrm{p}^{\mathrm{h}}$ & & $\mathrm{t}^{\mathrm{h}}$ & $\mathrm{c} \mathrm{c}^{\mathrm{h}}$ & $\mathrm{k} \mathrm{k}^{\mathrm{h}}$ & \\
\hline Nasal & $\mathrm{m} \mathrm{m}$ & & $\mathrm{n} \mathrm{n}$ & $\mathrm{n} \mathrm{n}$ & $\mathrm{n} \mathrm{g}$ & \\
\hline Enge & & $\mathrm{f} \mathrm{v}$ & $0 \mathrm{~g}$ & $\mathrm{c} \mathrm{j}$ & $\mathrm{x} \gamma$ & $\mathrm{h}$ \\
\hline Lateral & & & 11 & & & \\
\hline Vibrant & & & $\mathrm{r} \mathrm{r}$ & & & \\
\hline
\end{tabular}

Für diese Konsonanten geben wir folgende Beispiele:

Verschluß:

[p] bátur [pau:tyr] „Boot", bara [pa:ra] „nur“

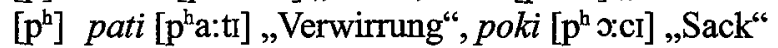

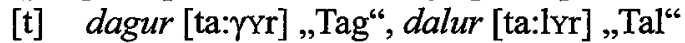

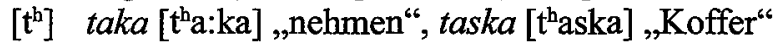

[c] geta [ce:ta] „können“, gjalda [calta],,Zahlen“

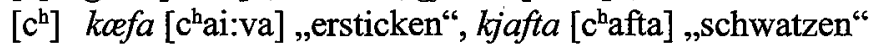

[k] gat [ka:t] „Loch“, gusa [kY:sa] „gießen“"

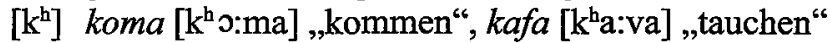

Nasal:

[m] hampa [hampa] „zeigen“, lampi [lampr] „Lampe“, cemta [aimta] „schreien“

[m] lamb [lamp] „Lamm“, mata [ma:ta] ,zum Essen geben“

[n] hné [nje:] ,Knie“, henta [henta] ,passen“"

[iْ] hanki [haujocr] „Haken“, banki [paujor] „Bank“

[ᄁ] hengi [heincr] „Behang“, lengja [leinca] ,verlängern“

[y] banka [paunka] „klopfen", vinka [vinka] „winken"

[n] langa [launka] „Lust haben“, Inga [inka] weiblicher Vorname

Enge:

[f] fara [fa:ra] „fahren“, sóffi [souf:I] „Sofa“ 
[v] vera [ve:ra] „sein“, vakta [vaxta] „bewachen“

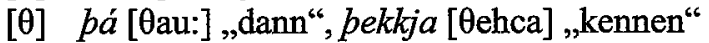

[ð] baða [pa:ða] „baden“, laða [la:ða] „anziehen“

[s] samur [sa:myr] „derselbe", vasi [va:sr] „Tasche“

[ç] hjá [çau] „bei“, hér [çe:r] „hier“

[j] já [jau:] ,ja“", segja [seija] „sagen“

[x] ekta [exta] „,echt", pekktur [0extyr] ,bekannt"

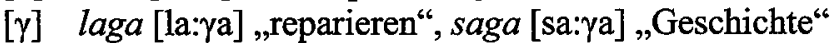

[h] hafa [ha:va] „haben“, hús [hu:s] „Haus“, petta [0ehta] „dieses“

\section{Lateral:}

[1] haltur [halttyr] „lahm“, hlaupa [löi:pa] „laufen“

[1] lafa [la:va] ,hängen“, halda [halta] ,halten“

Vibrant:

[r] hraði [ra:ðr] „Geschwindigkeit", marka [marka] „markieren“

[r] raða [ra:ða], ,ordnen“, meri [me:rI] „Stute“

\section{Lange Konsonanten:}

Nicht alle Konsonanten können lang sein. Lang können Konsonanten nur intervokalisch und final sein. Die folgenden Beispiele zeigen Paare von langen und kurzen Konsonanten in intervokalischer und finaler Stellung:

[p p:] tapa [ $\left.\mathrm{t}^{\mathrm{h}} \mathrm{a}: \mathrm{pa}\right]$,,verlieren“, rabba [rap:a] „,klönen“ tap [ $\left.\mathrm{t}^{\mathrm{h}} \mathrm{ap}\right]$ „Verlust", rabb [rap:] „Unterhaltung"

[t t:] mata [ma:ta] „zum Essen bringen", saddur [sat:Yr] ,gesättigt" mát [mau:t] „Matt“, rödd [rœet:] „Stimme“

[c c:] vekja [ve:ca] „wecken", leggja [lec:a] ,hinlegen“

[k k:] raka [ra:ka] „rasieren“, vagga [vak:a] „Wiege“ rök [rœ:k] „Argumente“, sigg [skk:] „Schwiele“

[m m:] hamast [ha:mast] „,sich betätigen“, komma $\left[\mathrm{k}^{\mathrm{b}} \mathrm{m}: \mathrm{a}\right]$ „Komma“ löm [lœm] ,Zarge“, skömm [skœm:] „Schande“

[n n:] hani [ha:nr] „Hahn“, sanna [san:a] „,beweisen“ lán [lau:n] „Glück“, brunn [pryn:] „Brunnen“" (Akk.Sing. von brunnur)

[f:] sóffi [souf:I] ,Sofa“"

[s s:] masa [ma:sa] „schwätzen“, massi [mas:I] „Masse“ hús [hu:s] „Haus“, húss [hus:] „Haus“ (Gen.Sing.)

[1 1:] mala [ma:la] „mahlen“, Elli [el:i] weiblicher Vorname, Elli [el:I] männlicher Vorname mal [ma:1] ,,(das) Schnurren“, ball [pal:] „Ball“

[r r:] meri [me:rI] „Stute“, verri [ver:I] „schlimmer“" her [he:r] „Armee“, verr [ver:] „schlimmer“ (Adv.)

Andere Konsonanten kommen nicht lang vor. Selbst unter den Konsonanten, die lang vorkommen können, gibt es Einschränkungen: 
1. Die palatalen [c c:] können kaum im Auslaut vorkommen. Es gibt wohl geggj „Verrücktheit“" [cec:] als einziges Wort mit langem Palatal im Auslaut.

2. Das lange [1:] kommt im Auslaut nur in wenigen Vornamen und Fremdwörtern vor.

Auffallend ist, $\mathrm{da} \beta \mathrm{im}$ Isländischen die aspirierten Verschlußkonsonanten nicht lang sein können. Von den Frikativen kommen nur [s] häufig und [f] selten in einigen Lehnwörtern und Wörtern lautmalerischer Art lang vor. Dagegen existiert das kurze [f] nicht intervokalisch. Ausnahmen können jedoch in Eigennamen vorkommen, z.B. Zóphanias [so:fa:nias]. Der stimmlose Lateral und der stimmlose Vibrant können nicht lang vorkommen. Daraus kann man ersehen, daß die Konsonantenquantität einen eingeschränkten Charakter im isländischen Konsonantensystem hat. Vielleicht erklärt sich daraus die in der Umgangssprache beobachtete Tendenz, die Konsonantenquantität abzubauen.

Ein Deutschsprechender, der Isländisch lernen will, muß sehr auf die Konsonantenquantität achten, da das Deutsche überhaupt keine langen Konsonanten besitzt.

\section{LAUT-SCHRIFT-BEZIEHUNGEN}

Die isländische Orthographie ist in ihrer Wesensart morphophonologisch und folgt Prinzipien, die der bekannte Sprachwissenschaftler Rasmus Kristjan Rask (17871832), der ein ausgezeichneter Kenner des Isländischen war, seinerzeit Anfang des 19. Jahrhunderts formuliert hatte. Vorwiegendes Ziel der Orthographie ist es danach, den Zusammenhalt der Sprache im Verlauf der Geschichte zu garantieren. Dies ist erstaunlich gut gelungen, denn isländische Sprecher können heute noch ohne vorheriges Studium die klassische Prosa-Literatur des 12. und 13. Jahrhunderts fließend und mühelos lesen. Selbst ein transliterierter Runentext ist nicht so sehr weit von der Sprache der Gegenwart entfernt, daß er mit relativ wenig Mühe gelesen und verstanden werden kann.

Die konservative Orthographie verschleiert die Tatsache, daß im Verlauf der Sprachgeschichte des Isländischen sehr bedeutsame und weitreichende Lautveränderungen stattgefunden haben. Die Folge davon ist, daß die jetzige Orthographie nach meiner Einschätzung nur zu etwa 50\% die tatsächliche Aussprache wiedergibt. Viele Elemente der Aussprache werden entweder gar nicht orthograpisch bezeichnet oder nur auf indirektem Wege. Dagegen bezeichnet die Orthographie Unterscheidungen, die in der modernen Sprache überhaupt nicht vorhanden sind und vor mehreren Jahrhunderten verschwanden. Beispiel hierfür sind die Buchstaben (Grapheme) $<y$ ý $>$, die ursprünglich hohe gerundete vordere Vokale [y y:] bezeichneten, die aber heute [I i] bezeichnen. Es gibt nur einen Buchstaben, nämlich $\langle\mathbf{p}\rangle$, der immer den gleichen Laut $[\theta]$ bezeichnet. Alle anderen Buchstaben sind mindestens zweideutig oder sogar mehrdeutig. Aus diesem Grunde sind die Laut-Schrift-Beziehungen im modernen Isländischen sehr komplex und das Erlernen der Orthographie erfordert eine große Anstrengung. Im Folgenden sollen nun diese wichtigsten Beziehungen kurz dargestellt werden.

\subsection{Konsonanten}

1. Das Graphem $<b>$ ist in den meisten Stellungen ein stimmloses [p], bara [pa:ra] „nur“. Es kann auch lang sein [p:] labba [lap:a] „gehen“. 
2. Das Graphem $<\mathrm{p}>$ ist aspiriert im Silbenanlaut poki [ $\left.\mathrm{p}^{\mathrm{h}} \mathrm{s}: \mathrm{cl}\right]$, Sack $^{c /}$, aber in anderen Stellungen [p] spara [spa:ra] „sparen“, sápa [sau:pa] „Seife. Vor einem anderen Verschlußlaut wird es zu [f] keypti [ceiftr] ,(ich) kaufte". Wird es <pp $>$ geschrieben, wird [hp] gesprochen: seppi [sehpr] „Hund“. Vor den Gruppen <pn pl> wird ebenfalls ein [h] eingeschoben: epli [ehpli] „Apfel“, heppni [hehpni] „Glück".

3. Das Graphem $<m>$ ist vor den graphischen $\langle p|>$ ein stimmloses [m], in anderen Stellungen hingegen ein stimmhaftes [m]: mega [mei:ya] „dürfen“, sama [sa:ma] „das gleiche“; hampur [hampyr] „Hanf“, skammta [skamta] „rationieren“".

4. Das Graphem $<\mathrm{f}>$ ist [f] im Anlaut: fara [fa:ra] „fahren“. Im Inlaut, Auslaut und vor $\langle\partial\rangle$ hingegen ist es [v]: sofa [so:va] ,schlafen", of [o:v] ,zuviel", sof $\partial u$ [s əvठy] „,schlaf" (Imperativ). In den Gruppen <fl fn> ist es [p]: efni [epnr] ,Stoff“, efla [epla] „,befördern“.

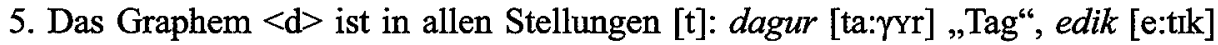
„Essig“. Wenn es <dd> geschrieben wird, ist es [t:]: Edda [et:a] weiblicher Vorname.

6. Das Graphem $<t>$ ist im Silbenanlaut aspiriert [ $\left.\mathrm{t}^{\mathrm{h}}\right]$ : taka [ $\left.\mathrm{t}^{\mathrm{h}} \mathrm{a}: \mathrm{ka}\right]$,"nehmen ${ }^{\text {cc }}$. In allen anderen Stellungen ist es unaspiriert [t]: stara [sta:ra] „starren“, gata [ka:ta] „Straße".

In den orthographischen Gruppen <ll $\mathrm{nn} \mathrm{rl} \mathrm{m}>$ wird es überhaupt nicht geschrieben: allir [atlir] „alle“, Árni [autn]] Eigenname, varla [vatla] „kaum“, seinna [seitna] „später" (Adv. Komparativ).

Die orthographischen Gruppen < tt $\mathrm{tl} \mathrm{tn}>$ haben ein [h] vor dem Verschlußlaut: hattur [hahtyr] „Hut", atla [aihtla] „vorhaben", vatn [vahtn] „Wasser“.

7. Das Graphem $<\mathrm{n}>$ ist intervokalisch und im Anlaut stimmhaft. Stimmlos ist es im Inlaut vor einem Graphem $<\mathrm{t} k>$, im Auslaut nach stimmlosen Konsonanten und im Anlaut, wenn es <hn> geschrieben wird: ná [nau:] „erreichen“, hani [ha:nI] „Hahn“, henta [hetnta] „passen“, einn [eitn] „ein“, hnifur [ni:vyr] „Messer“. Vor dem Graphem <d> ist es stimmhaft [n]: henda [henta] ,werfen". Wenn es <nn> geschrieben wird, ist es entweder lang [n:] oder [tn tn]: hanna [han:a] „entwerfen“, voenna [vaitna] „lieber" (Adv. Komparativ), hreinn [reitn] „sauber“.

Vor den Graphemen $<\mathrm{g} \mathrm{k}>$ ist $<\mathrm{n}>$ entweder palataler oder velarer Nasal, je nach der Artikulationsstelle des nachfolgenden Konsonanten, stimmlos vor $<\mathrm{k}>$, stimmhaft vor <g>: langa [launga] ,wünschen", hanki [haục cI] „Haken", hringja [riyca]“anrufen“, vinka [vị̧ka] ,winken“.

8. Das Graphem <s> ist immer apiko-alveolar, [s], entweder lang oder kurz: masa [ma:sa] „schwätzen", massi [mas:I] „Masse".

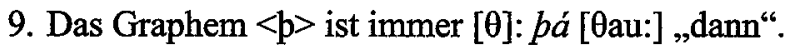


10. Das Graphem $<\chi>$ ist [ð], aber im Inlaut vor $<\mathrm{k}>$ ist es [ $\theta]$ : baða [pa:ða] ,baden“, maðkur [ma日krr] „Wurm“.

11. Das Graphem $<\mathrm{l}>$ ist stimmhaft im Anlaut, intervokalisch und vor allen Konsonanten außer <p t k>: ala [a:la] ,ernähren“, aldur [altyr] „Alter", val [va:1] „Wahl, Selektion“.

Wenn es <ll> geschrieben wird, kann es lang sein [1:] oder die Gruppen [tl tl] darstellen: villa [vil:a] „Villa“, villa [vitla] „Fehler“, höll [hœetl] „Schloß“.

Die Gruppe <hl> wird als stimmloses [1] gesprochen: hlé [l je:] „Unterbrechung“. Vor den Graphemen $<\mathrm{p}$ t $\mathrm{k}>$ ist $<\mathrm{l}>$ stimmlos [1] : hjálpa [çaulpa] ,helfen“, valtur [valtyr] ,instabil“, kalk [k $\left.\mathrm{k}_{\mathrm{al}} \mathrm{k}\right]$,Kalk“.

12. Das Graphem $<r>$ ist immer eine apiko-alveolare Vibrante, in den meisten Stellungen stimmhaft [r]: rá [rau:] „Rabe“. Wenn es <rr> geschrieben wird, ist es intervokalisch und im Auslaut lang [r:]: kerra [cher:a] „Karre“, verr [ver:] „schlimmer" (Adv. Komparativ).

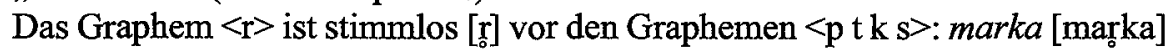
„markieren“, varta [varta] „Warze“, harka [harka] „Härte“, sársauki [saursœicr] „Schmerz". Die Graphemfolge <hr> im Anlaut wird [r] gesprochen: hrár [rau:r] „roh“.

13. Das Graphem $<\mathrm{g}>$ ist im Anlaut vor den Graphemen $<$ ö u a á au ó o ú $>[\mathrm{k}]$ oder langes [k:] im Inlaut, wenn es <gg> geschrieben wird: gata [ka:ta] „Gasse“, vagga [vak:a] „Wiege“.

Die Graphemfolge <gj> bezeichnet den palatalen Verschlußlaut [c], und <ggj> den langen Palatalen [c:]: gjalda [calta] ,zahlen“, leggja [lec:a] „,hinlegen“. Das Graphem $<\mathrm{g}>$ wird vor den Graphemen $<\mathrm{i}$ í y ý æ e ei ey> palatal [c] gesprochen: gir [ci:r] „Schaltung“, goes [cai:s] „Gans“.

Das $<\mathrm{g}>$ wird intervokalisch vor gerundeten Vokalen und vor [a], im Auslaut nach Vokal und im Inlaut vor $<ð>$ als stimmhafte Frikative $[\gamma]$ gesprochen: laga

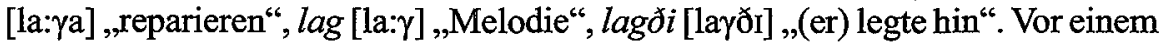
$<\mathrm{t}>$ wird es [x] gesprochen: sagt [saxt] ,gesagt", lagt [laxt] ,gelegt“.

Wenn <gj> intervokalisch vorkommt, ist es [j]: segja [sei:ja] ,sagen“. Intervokalisches $<\mathrm{g}>$ vor vorderen ungerundeten Vokalen ist ebenfalls [j]: lögin [lœi:jin] „die Gesetze“.

14. Das Graphem $<\mathrm{k}>$ ist aspiriert im Silbenanlaut, palatal wenn es $<\mathrm{kj}>$ geschrieben wird und vor den Graphemen <i í y ý æ e ei ey>, aber in anderen Fällen velar:

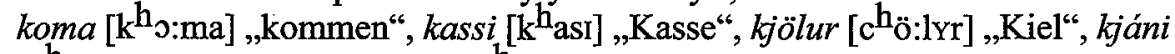

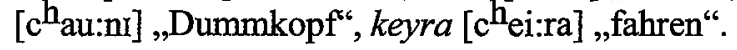

Vor einem Konsonanten [t] oder [s] wird es [x] gesprochen: ekta [exta] „echt", feykt [feixt] ,gefegt", loks [10xs] ,schließlich“. Die Graphemfolgen <kk kkj kkl $\mathrm{kl} \mathrm{kn}>$ werden mit einem [h] vor dem Verschlußlaut gesprochen: hakka [habka] „hacken", ekkja [ehca] „Witwe“, ökkli [œhklr] „Fußgelenk“, lyklar [lihklar] „Schlüssel“ (P1.), vökna [vœhkna] ,naß werden“". 
15. Das Graphem $<\mathrm{x}>$ bezeichnet die Lautfolge [xs]: lax [laxs] „Lachs“.

16. Das Graphem $<\mathrm{h}>$ wird im Anlaut [h] ausgesprochen: hüfa [hu:va] „Mütze“. Im Inlaut vor einem Konsonanten wird es überhaupt nicht geschrieben. Die Graphemfolgen $\langle\mathrm{hr} \mathrm{hl} \mathrm{hn} \mathrm{hj>} \mathrm{werden} \mathrm{[} \mathrm{r} l \mathrm{n}$ ç] ausgesprochen. Vor $<\dot{e}>$ wird $<\mathrm{h}>$ als [ç] realisiert: héri [çe:rr] „Hasen“. Die Graphemfolge $<\mathrm{hv}>$ ist $\left[\mathrm{k}^{\mathrm{h}} \mathrm{v}\right.$ ]: hvar

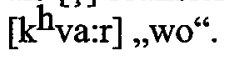

\subsection{Vokale und Diphtonge}

Die Laut-Schrift-Beziehungen sind bei Vokalen und Diphthongen etwas einfacher als bei den Konsonanten. Allerdings sind sie auch nicht ganz regelmäßig, so daß auf einiges geachtet werden muß. Das Akzentzeichen $\langle>>$ über einigen Vokalen bezeichnet keine Betonung, sondern je nach Vokal eine bestimmte Klangfarbe bzw. einen Diphthong. Wie schon erwähnt wurde (s. 2.1.), ist die Betonung aller Wörter im Isländischen ausnahmslos auf der ersten Silbe.

Im folgenden werden die Laut-Schrift-Beziehungen für Vokale und Diphthonge kurz zusammengefaßt:

\begin{tabular}{|c|c|c|c|}
\hline \multicolumn{3}{|c|}{ entsprechen die Grapheme } & 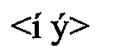 \\
\hline$"$ & $"$ & $"$ & $<i y>$ \\
\hline & & " & $<$ e é $>$ \\
\hline \multicolumn{3}{|c|}{ entspricht das Graphem } & $<\mathbf{u}>$ \\
\hline$"$ & $"$ & " & $<\ddot{0}>$ \\
\hline " & $"$ & " & $<\dot{\mathbf{u}}>$ \\
\hline " & $"$ & " & $<0>$ \\
\hline & & $"$ & $<\mathrm{a}>$ \\
\hline \multirow{2}{*}{\multicolumn{3}{|c|}{$\begin{array}{l}\text { entsprechen die Grapheme } \\
\text { entspricht das Graphem }\end{array}$}} & $<$ <i ey> \\
\hline & & & $<\mathrm{au}>$ \\
\hline$"$ & $"$ & $"$ & $<\mathfrak{x}\rangle$ \\
\hline$"$ & $"$ & $"$ & $<\dot{a}>$ \\
\hline$"$ & $"$ & $"$ & $<\hat{o}>$ \\
\hline
\end{tabular}

$\mathrm{Zu}$ diesen Entsprechungen gibt es einige wenige Ausnahmen, wobei die wichtigsten folgende sind:

1. Vor der Graphemfolge <ng nk> werden die Vokale wie folgt ausgesprochen:

\begin{tabular}{|c|c|c|c|}
\hline als & {$[\mathrm{au}]$} & langur [launkYr] & "lang“ \\
\hline als & [i] & hringur [rrijkyr] & „Ring“ \\
\hline als & [ei] & lengur [leinkYr] & „länger"c \\
\hline 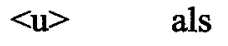 & {$[\mathrm{u}]$} & ungur [unkrr] & ,jung“c \\
\hline als & [œi] & bröng [Orœink] & "Enge“" \\
\hline
\end{tabular}

Hierzu gibt es wiederum nur ganz wenige Ausnahmen wie z.B. vangá [vankau] "Unachtsamkeit".

2. In einigen sehr häufigen Wörtern wird $<y>$ häufig als $[\mathrm{Y}]$ ausgesprochen (nicht als [I] oder nur selten): spyrja [spyrja] „fragen“, ykkur [Yhkyr] „euch“. 
3. In seltenen Fällen wird $<\mathrm{i}>$ als [i] ausgesprochen: illur [itlyr] „schlimm“.

4. <é> ist die Lautfolge [je]: él [je:1] „Schneeschauer“.

\section{HINWEISE FÜR DEN UNTERRICHT}

Der phonetische Aspekt im Fremsprachenunterricht ist in Island bisher stark vernachlässigt worden. Zur Zeit existiert kaum Lehrmaterial, das für den Ausspracheunterricht konzipiert oder geeignet wäre. Das könnte sich aber bald ändern, da die große Zahl von Immigranten in Island und die Probleme ihrer Eingliederung in die Gesellschaft die Aufmerksamkeit der Schulbehörde geweckt haben.

Die Erfahrung hat gezeigt, daß unter den Einwanderern in Island die Deutschen die isländische Aussprache relativ leicht lernen. Viele dort lebende Deutsche sprechen Isländisch fast perfekt, sowohl phonetisch als auch grammatisch. Dies ist möglich, ohne daß man dort geboren sein muß. Ein Rezept für gute Aussprache gibt es wohl nicht. Es kommt sehr viel auf die Motivation und die Nachahmungsfähigkeit des Einzelnen an.

Im folgenden soll nur kurz darauf hingewiesen werden, wo erfahrungsgemäß die größten Problembereiche liegen:

1. Das isländische Vokalsystem bietet für Deutsche kaum Probleme. Der Vokal [Y] hat im Deutschen allerdings keine genaue Entsprechung und darauf muß anfangs eine gewisse Aufmerksamkeit gerichtet sein. Auch ist es für einen Deutschen fremd, $\mathrm{da} \beta[\mathrm{I}]$ und [0] im Isländischen lang sein können, was im Deutschen bekanntlich nicht der Fall ist. Diese Vokale können im Deutschen nur kurz vorkommen.

2. Im Konsonantensystem sind die stimmlosen Nasale [mo im allgemeinen das schwierigste Problem für alle Ausländer. Schwierig ist auch das [h], insbesondere im Wortinnern vor einem Verschlußlaut: ekki [ehcr] ,nicht“". Auch die palatalen Verschlußlaute bereiten anfangs gewisse Schwierigkeiten.

3. Deutsche müssen besonders darauf achten, daß im Isländischen kein stimmhaftes $\mathrm{s}$ [z] wie in Sohn [zo:n] existiert. Das isländische <s> ist immer apiko-alveolar und stimmlos [s].

Umgekehrt haben Isländer, die Deutsch lernen, das größte Problem mit den Konsonanten [z] und [j], die im Isländischen nicht vorhanden sind. Im Vokalsystem bereitet das Schwa [ə] die größten Probleme. Dagegen ist das [y] kaum problematisch, obwohl es im Isländischen nicht existiert. Das uvulare [R] und das vokalisierte $\langle\mathbf{r}>[\mathrm{e}]$ des Deutschen bereiten Isländischsprechenden gewisse Schwierigkeiten beim Lernen der deutschen Aussprache.

Prosodisch bereitet der bewegliche Akzent des Deutschen, d.h. die Tatsache, daß der deutsche Akzent nicht immer auf die erste Silbe fällt, den Isländern große Schwierigkeiten. Darauf sollte in den Anfangsstufen des Unterrichts besonders geachtet werden.

\section{WEITERFÜHRENDE LITERATUR}

Die folgende Literatur gibt die im Artikel zitierten Werke an und außerdem einige Werke zu verschiedenen Aspekten der isländischen Sprache und der isländischen Kultur. Werke, die sich besonders auf die Aussprache beziehen, gibt es kaum, u.a. kein Aussprachewörterbuch. 
DUPPLER, Ríta und Nahl, Astrid von: Langenscheidts Universal-Wörterbuch: Isländisch (Langenscheidt, Berlin 1993)

ELLERTSSON, Björn: Íslensk-pýsk orðabók; Isländisch-deutsches Wörterbuch (Iðunn, Reykjavík 1993)

GRÖNKE, Ulrich: Diachrone Perdurabilität, Sprachpflege und Sprachplanung: Der Fall Isländisch. In: István Fodor and Claude Hagège (eds.), Language Reform, History and Future, Vol. II, pp. 137-155 (Helmut Buske Verlag, Hamburg 1983)

JơRG, Christine: Isländische Konjugationstabellen (Helmut Buske Verlag, Hamburg 1989)

KRESS, Bruno: Isländische Grammatik (VEB Verlag Enzyklopädie, Leipzig; Max Hueber Verlag, München 1982)

KRISTJÁNSSON, Jónas: Eddas und Sagas: Die mittelalterliche Literatur Islands (Helmut Buske Verlag, Hamburg 1994)

Nordat, Jóhannes and Kristinsson, Valdimar (eds.): Iceland 874-1974 (The Central Bank of Iceland, Reykjavík 1975)

ÓFErGSSON, Jón: Býzk-íslenzk orðabók (Ísafoldarprentsmiðjja, Reykjavik 1953)

PÉtursson, Magnús: La préaspiration en islandais. Examen de sa réalisation phonétique chez deux sujets. Studia Linguistica 26, 61-80 (1972)

PÉtursson, Magnús: Les articulations de l'islandais à la lumière de la radiocinématographie (Klincksieck, Paris 1974)

Pétursson, Magnús: Drög að almennri og íslenskri hljóðfræði (Iðunn, Reykjavík 1976)

PÉtursson, Magnús: Isländisch (Helmut Buske Verlag, Hamburg 1978)

Pétursson, Magnús: Lehrbuch der isländischen Sprache mit Übungen und Lösungen (Helmut Buske Verlag, Hamburg 2006; 4. Auflage)

Selting, Margret: Prosodie im Gespräch. Aspekte einer interaktionalen Phonologie der Konversation (Niemeyer, Tübingen 1995)

THOMSON, Colin D.: İslensk beygingafræðð; Isländische Formenlehre; Icelandic Inflections (Helmut Buske Verlag, Hamburg.1987)

\section{Povzetek}

\section{PODLAGE ZA FONETIČNE VAJE POD VODSTVOM ROJENIH GOVORCEV ISLANDŠC̆INE}

Po uvodnih pojasnilih o zgodovini in sedanjih jezikovnih razmerah na Islandskem podaja avtor naslednje preglede: o splošnih značilnostih islandščine, o suprasegmentalnih pojavih (naglas, intonacija, ritmika), o segmentalnih pojavih, in sicer o zgradbi zloga, o samoglasnikih (o enoglasnikih in dvoglasnikih), o soglasnikih, o razmerju med izgovorjavo in pisavo. Na koncu so praktična navodila za pouk o islandski izgovorjavi. 